\title{
Transverse instability and dynamics of nonlocal bright solitons
}

\author{
G. N. Koutsokostas,${ }^{1}$ G. Theocharis,${ }^{2}$ T. P. Horikis,${ }^{3}$ P. G. Kevrekidis,${ }^{4}$ and D. J. Frantzeskakis ${ }^{1}$ \\ ${ }^{1}$ Department of Physics, National and Kapodistrian University of Athens, \\ Panepistimiopolis, Zografos, Athens 15784, Greece \\ ${ }^{2}$ LAUM, CNRS, Le Mans Université, Avenue Olivier Messiaen, 72085 Le Mans, France \\ ${ }^{3}$ Department of Mathematics, University of Ioannina, Ioannina 45110, Greece \\ ${ }^{4}$ Department of Mathematics and Statistics, University of Massachusetts, Amherst, MA 01003-4515, USA
}

\begin{abstract}
We study the transverse instability and dynamics of bright soliton stripes in two-dimensional nonlocal nonlinear media. Using a multiscale perturbation method, we derive analytically the firstorder correction to the soliton shape, which features an exponential growth in time - a signature of the transverse instability. The soliton's characteristic timescale associated with its exponential growth, is found to depend on the square root of the nonlocality parameter. This, in turn, highlights the nonlocality-induced suppression of the transverse instability. Our analytical predictions are corroborated by direct numerical simulations, with the analytical results being in good agreement with the numerical ones.
\end{abstract}

Keywords:

\section{INTRODUCTION}

Wave instabilities play an important role in the evolution of nonlinear systems, as they are associated with symmetry breaking effects resulting in the formation of coherent structures or leading to chaotic states [1]. A pertinent example is the modulational instability (MI) of plane waves in media governed by the focusing nonlinear Schrödinger (NLS) equation, with MI resulting in a variety of important nonlinear processes, such as the formation of envelope bright solitons, envelope shock waves, and rogue waves [2]. Another example is the transverse instability, which refers to the growth of transverse modulations of quasi one-dimensional (1D) (stripe) bright and dark solitons, for focusing [3, 4] and defocusing [5, 6] NLS models, respectively. In the elliptic 2D focusing NLS, the norm of the soliton stripes is infinite, as they extend to infinity in the transverse direction. Hence, the bright soliton stripes, being subject to the onset of collapse, break up, through a width modulation, into individual $2 \mathrm{D}$ lump-shaped structures. In such a case, the transverse instability is of the so-called "necking" type [7]. On the other hand, bright solitons of the hyperbolic 2D focusing NLS, as well as dark solitons of the elliptic $2 \mathrm{D}$ defocusing NLS, undergo undulations of the location of their center, due to the transverse instability, and eventually decay; due to this, so-called "snaking" instability, bright soliton stripes decay into bright lumps, while dark solitons decay into vortices or dark lumps [8, 9]. It is important to note that this is a popular experimental technique for observing the instability outcome and subsequent pattern formation both in atomic physics 10] and in nonlinear optics [11, 12].

Arrest or substantial suppression of the transverse instability of solitons has been proved to be a topic of great interest, and various physical mechanisms have been proposed to suppress this instability. These mechanisms include the coupling of solitons with another soliton component [13, 14], making the soliton sufficiently incoher- ent along the transverse direction 15], as well as using periodic lattice potentials [16, 17] or localized barrier potentials [18]. In addition, nonlocal nonlinearities, occurring e.g., in plasmas [19], atomic vapors [20], lead glasses 21], nematic liquid crystals 22], as well as dipolar BoseEinstein condensates [23], are known to play a key role on the stability of solitons. In particular, in settings with focusing nonlocal nonlinearities, the transverse instability of bright nonlocal solitons can be substantially suppressed [24], while collapse can be arrested in higherdimensions 25, 26] and, as a result, stable 2D and 3D solitons can be formed 20, 21, 26 28]. On the other hand, in settings with defocusing nonlocal nonlinearities, the transverse instability of dark nonlocal solitons $29-32$. can be suppressed [33].

In this work, we revisit the problem of the transverse instability and dynamics of bright solitons in nonlocal nonlinear media. The considered nonlocal NLS model, namely a Schrödinger type paraxial wave equation, coupled with a diffusion-type equation governing the nonlocal response of the medium, is relevant to a variety of physical contexts. These include optical media with a thermal nonlinearity (e.g., atomic vapors [20, 21] and liquid solutions 34, 35]), plasmas 36, 37], and nematic liquid crystals [38, 39]. The considered nonlocal NLS possesses a $\operatorname{sech}^{2}$-shaped exact analytical bright soliton solution, which can not be reduced - in the local nonlinearity limit - to the usual sech-shaped bright soliton of the NLS; hence, one can not exploit this limit to study the effect of nonlocality on the transverse instability of nonlocal soliton stripes, as has been done, e.g., in [24].

Here, we analyze the problem by employing a perturbation method, similar to the one used in Ref. 40] (see also [1]), which uses a perturbation ansatz relying on the $\mathrm{sech}^{2}$ nonlocal soliton, with a center and a phase becoming unknown functions of slow time and transverse coordinate. We find an approximate solution of the nonlocal NLS, where the correction to the soliton shape is shown to feature an exponential growth in time, which is a sig- 
nature of the transverse instability. The latter is of the necking type, and is induced by the soliton phase, which is shown to obey an elliptic partial differential equation (PDE). We show that the instability growth rate (i.e., the inverse characteristic time scale associated with the exponential growth of the phase), depends on the inverse square root of the nonlocality parameter, a fact highlighting the substantial, nonlocality-induced suppression of the transverse instability of the bright soliton stripes (in a similar vein as earlier works [24]). The analytical estimation for the growth rate, as well as the derived approximate analytical solution, are found to be in good agreement with respective results obtained by means of direct simulations. Despite the prolongation of the lifetime of the solutions obtained herein, our results do not support the scenario of a complete stabilization of the relevant bright soliton stripes, irrespectively of the value of nonlocality parameter $\nu$, for the range considered herein.

The presentation of the manuscript is organized as follows. In Section II, we introduce the model and its exact soliton solution, and present the results of our perturbation method; these include the derivation of the evolution of the soliton parameters, the derivation of the instability growth rate, as well as the first-order correction of the soliton shape. Section III is devoted to the presentation of our numerical results, and comparison with the analytical approximations. Finally, in Section IV we summarize our conclusions and discuss possibilities for relevant future research.

\section{MODEL AND STABILITY ANALYSIS}

\section{A. The model and its exact $1 \mathrm{D}$ soliton solutions}

We consider the propagation of an optical beam in a nonlocal nonlinear medium. Let $u$ be the complex electric field envelope of the light beam satisfying a paraxial, Schrödinger-type equation, and the real function $\theta$ be the nonlinear, generally nonlocal, medium's response, assumed to obey a diffusion-type equation [28]. Then, the evolution of the beam is governed by the following dimensionless nonlocal NLS model:

$$
\begin{aligned}
i u_{t}+\frac{d}{2} \Delta u+2 g \theta u & =0, \\
\nu \Delta \theta-2 q \theta+2 g|u|^{2} & =0,
\end{aligned}
$$

where subscripts denote partial derivatives. Here, the evolution variable $t$ represents the propagation distance (assumed to be along the $z$ direction), $\Delta \equiv \partial_{x}^{2}+\partial_{y}^{2}$ is the transverse Laplacian, while $g$ and $d$ are coupling and diffraction coefficients, assumed to be positive; this case corresponds to a focusing nonlinearity. In addition, $q>0$ is a constant and, finally, the parameter $\nu$, which measures the diffusion length (assumed to be large compared to the operating wavelength), describes the strength of nonlocality: indeed, large $\nu$ corresponds to a highly nonlocal response while in the limit $\nu \rightarrow 0$, Eqs. (11)-(2) re- duce to the following NLS equation with a local cubic (Kerr-type) nonlinearity:

$$
i u_{t}+\frac{d}{2} \Delta u+\frac{2 g^{2}}{q}|u|^{2} u=0 .
$$

The model (11)-(2) is relevant to a variety of nonlocal media. These include: (a) optical media featuring a thermal nonlinearity - such as atomic vapors [20, 21] and liquid solutions, with $\theta$ being the nonlinear correction to the refractive index [34, 35]; (b) ionized plasmas, with $\theta$ being the relative electron temperature perturbation, and $q \propto m / M$ being the relative energy that an electron of mass $m$ delivers to a heavy particle of mass $M$ during a single collision [36, 37]; (c) nematic liquid crystals [38, 39], with $\theta$ denoting the perturbation of the optical director angle from its static value due to the light beam, and $q$ being related to the applied static field which pretilts the nematic dielectric [22, 42].

As explained in the Introduction, our scope is to study the transverse dynamics of 1D bright soliton stripes in the $2 \mathrm{D}$ setting and investigate, in particular, the role of nonlocality. It is thus convenient to start by presenting such $1 \mathrm{D}$ bright soliton solutions of Eqs. (1-2), which can be found upon using the ansatz [43]:

$$
u=q_{0}(\xi) \exp \left[i \omega\left(t+\sigma_{0}\right)\right], \quad \theta=\theta_{0}(\xi),
$$

where $q_{0}$ is an unknown real function depending on $\xi=k\left(x-x_{0}\right), k$ is an unknown constant, $\omega$ is the unknown frequency of the solution, while $x_{0}$ and $\sigma_{0}$ are arbitrary real parameters representing, respectively, the initial location and the phase of the soliton. Substituting Eqs. (4) into Eqs. (11)-(2), it can be found that the resulting equations become:

$$
\begin{aligned}
d k^{2} q_{0 \xi \xi}-2 \omega q_{0}+4 g q_{0} \theta_{0} & =0 \\
\nu k^{2} \theta_{0 \xi \xi}-2 q \theta_{0}+2 g q_{0}^{2} & =0 .
\end{aligned}
$$

Then, observing that if

$$
\theta_{0}=\sqrt{\frac{d}{2 \nu}} q_{0}, \quad \text { and } \quad \omega=\frac{d q}{\nu},
$$

then the system (5)-(6) reduces to a single ordinary differential equation (ODE):

$$
q_{0 \xi \xi}-\frac{2 g}{\nu k^{2}} q_{0}+\frac{4 g}{\sqrt{2 \nu d} k^{2}} q_{0}^{2}=0 .
$$

The latter possesses the exact soliton solution

$$
q_{0}(\xi)=\frac{3 q}{2 g} \sqrt{\frac{d}{2 \nu}} \operatorname{sech}^{2}\left[k\left(x-x_{0}\right)\right], \quad k=\sqrt{\frac{q}{2 \nu}},
$$

which implies that the soliton solutions of Eqs. (11)-(2) are of form:

$$
\begin{aligned}
& u_{0}(x, t)=\frac{3 q}{2 g} \sqrt{\frac{d}{2 \nu}} \operatorname{sech}^{2}\left[k\left(x-x_{0}\right)\right] \exp \left[i \omega\left(t+\sigma_{0}\right)\right],(10) \\
& \theta_{0}(x, t)=\frac{3 d q}{4 g \nu} \operatorname{sech}^{2}\left[k\left(x-x_{0}\right)\right] .
\end{aligned}
$$


Here it is interesting to note that, while the system of Eqs. (11)-(2) reduces to the local NLS (3), the exact solution (10) cannot be reduced to the soliton solution of Eq. (3) (which features a sech-profile and is characterized by a free parameter); this becomes clear by the fact that $\lim _{\nu \rightarrow 0} u=0$. It is also mentioned that Eqs. (10)-(11) represent a stationary solution of the problem; traveling solutions exist as well and can easily be constructed by means of a Galilean boost.

\section{B. Perturbation theory}

In order to study the stability of solutions (10)-(11) in two dimensions, we consider solutions of Eqs. (11)-(2) in the form of the following asymptotic expansions:

$$
\begin{aligned}
u\left(\xi, t, T_{i}, Y_{i}\right) & =\sum_{j=0}^{\infty} \epsilon^{j} q_{j}(\xi) \exp \left[i \omega\left(t+\sigma_{0}\left(T_{i}, Y_{i}\right)\right)\right] \\
\theta\left(\xi, T_{i}, Y_{i}\right) & =\sum_{j=0}^{\infty} \epsilon^{j} \theta_{j}\left(\xi, T_{i}, Y_{i}\right) \\
\xi & =k\left[x-x_{0}\left(T_{i}, Y_{i}\right)\right]
\end{aligned}
$$

where $T_{i}=\epsilon^{i} t, Y_{i}=\epsilon^{i} y$ and $0<\epsilon \ll 1$ is a formal small parameter. The above perturbation ansatz is actually inspired by the form of the exact solution (4) of the 1D problem but, now, with the soliton's center $x_{0}$ and phase $\sigma_{0}$ becoming unknown functions of the slow variables $T_{i}$ and $Y_{i}$. Substituting the perturbation expansions (12)(13) into Eqs. (11)-(2) we obtain the following results.

First, at $\mathcal{O}\left(\epsilon^{0}\right)$, we obtain the system (5)-(6), which provides the exact soliton solution (9) [that eventually leads, together with (7), to the solution of Eqs. (10)-(11)].

At the next orders of approximation, the presence of derivatives of $x_{0}$ and $\sigma_{0}$ with respect to the slow variables renders the inhomogeneous parts $F_{j}$ of the resulting equations for $q_{j}$ (with $j=1,2, \ldots$ ) complex, i.e., $F_{j}=F_{j}^{(\mathrm{r})}+i F_{j}^{(\mathrm{i})} ;$ this implies that $q_{j}$ itself must be complex, i.e., $q_{j}=q_{j}^{(\mathrm{r})}+i q_{j}^{(\mathrm{i})}$. Thus, separating real and imaginary parts of the resulting equations, we obtain, at each order, a set of three equations, two of which are coupled. To be more specific, the resulting equations at orders $\mathcal{O}\left(\epsilon^{j}\right)$ for $j=1,2, \ldots$ take the following form:

$$
\begin{aligned}
& \left(d k^{2} \partial_{\xi}^{2}-2 \omega+4 g \theta_{0}\right) q_{j}^{(\mathrm{r})}+4 g q_{0} \theta_{j}=F_{j}^{(\mathrm{r})} \\
& \left(d k^{2} \partial_{\xi}^{2}-2 \omega+4 g \theta_{0}\right) q_{j}^{(\mathrm{i})}=F_{j}^{(\mathrm{i})} \\
& \left(\nu k^{2} \partial_{\xi}^{2}-2 q\right) \theta_{j}+4 g q_{0} q_{j}^{(\mathrm{r})}=G_{j} .
\end{aligned}
$$

The inhomogeneous parts at the order $\mathcal{O}(\epsilon)$ are given by:

$$
F_{1}^{(\mathrm{r})}=2 \omega \sigma_{0 T_{1}} q_{0}, \quad F_{1}^{(\mathrm{i})}=2 k x_{0 T_{1}} q_{0 \xi}, \quad G_{1}=0,
$$

while at the order $\mathcal{O}\left(\epsilon^{2}\right)$ they read:

$$
\begin{aligned}
F_{2}^{(\mathrm{r})}= & -4 g q_{1}^{(\mathrm{r})} \theta_{1}-d k^{2} x_{0 Y_{1}}^{2} q_{0 \xi \xi}+d k x_{0 Y_{1} Y_{1}} q_{0 \xi} \\
& +d \omega^{2} \sigma_{0 Y_{1}}^{2} q_{0}+2 \omega \sigma_{0 T_{2}} q_{0}+2 \omega \sigma_{0 T_{1}} q_{1}^{(\mathrm{r})} \\
& +2 q_{1 T_{1}}^{(\mathrm{i})}-2 v x_{0 T_{1}} q_{1}^{(\mathrm{i})} \\
F_{2}^{(\mathrm{i})}= & -4 g q_{1}^{(\mathrm{i})} \theta_{1}+2 d k \omega x_{0 Y_{1}} \sigma_{0 Y_{1}} q_{0 \xi}-d \omega \sigma_{0 Y_{1} Y_{1}} q_{0} \\
& +2 k x_{0 T_{2}} q_{0 \xi}+2 \omega \sigma_{0 T_{1}} q_{1}^{(\mathrm{i})}-2 q_{1 T_{1}}^{(\mathrm{r})} \\
& +2 k x_{0 T_{1}} q_{1 \xi}^{(\mathrm{r})} \\
G_{2}= & -\nu k^{2} x_{0 Y_{1}}^{2} \theta_{0 \xi \xi}+\nu k x_{0 Y_{1} Y_{1}} \theta_{0 \xi} \\
& -2 g\left(q_{1}^{(\mathrm{r}) 2}+q_{1}^{(\mathrm{i}) 2}\right) .
\end{aligned}
$$

To proceed further, it is useful to make a few observations. First, differentiating Eqs. (5)-(6) with respect to $\xi$, one obtains the homogeneous part of Eqs. (15)-(17). This implies that the homogeneous solutions of Eqs. (15)-(17) are of the form:

$$
q_{j h}^{(\mathrm{r})}=q_{0 \xi}, \quad q_{j h}^{(\mathrm{i})}=q_{0}, \quad \theta_{j h}=\theta_{0 \xi} .
$$

Second, having found the above homogeneous solutions, we may derive the solvability conditions of the full inhomogeneous problem, Eqs. (15)-(17). To do this, first we consider the coupled Eqs. (15) and (17). We multiply both sides of Eqs. (15) by the homogeneous solution $q_{j h}^{(\mathrm{r})}$, as well as both sides of Eq. (17) by the homogeneous solution $\theta_{j h}$. Then, we add the resulting equations and integrate with respect to $\xi$ from $-\infty$ to $+\infty$. This yields the following integral relation:

$$
\int_{-\infty}^{\infty}\left(q_{j h}^{(\mathrm{r})} F_{j}^{(\mathrm{r})}+\theta_{j h} G_{j}\right) d \xi=0
$$

which is the solvability condition of Eqs. (15) and (17). To obtain the solvability condition for Eq. (16), we follow a similar procedure, namely we multiply both sides of Eq. (16) by the homogeneous solution $q_{j h}^{(\mathrm{i})}$ and integrate from $-\infty$ to $+\infty$; this yields:

$$
\int_{-\infty}^{\infty} q_{j h}^{(\mathrm{i})} F_{j}^{(\mathrm{i})} d \xi=0
$$

Importantly, the above solvability conditions will lead to evolution equations for the soliton center $x_{0}$ and phase $\sigma_{0}$ which - as we will see - will provide the necessary information for characterizing the stability of the 1D soliton solutions. Furthermore, solving Eqs. (15)-(17) (for $j=1$ ) will provide us the form of the solution to Eqs. (1)-(2) up to $\mathcal{O}(\epsilon)$, and for short times - up to the onset of the instability. This will be particularly relevant for our direct numerical simulations as well.

\section{Evolution of the soliton parameters}

First we consider the problem at the order $\mathcal{O}(\epsilon)$. In this case, the solvability conditions, Eqs. (23) and (24), 
lead to the following results, respectively:

$$
\int_{-\infty}^{\infty} q_{0 \xi}\left(2 \omega \sigma_{0 T_{1}} q_{0}\right) d \xi=2 \omega \sigma_{0 T_{1}} \int_{-\infty}^{\infty}\left(q_{0} q_{0 \xi}\right) d \xi=0
$$

and

$$
\int_{-\infty}^{\infty} q_{0}\left(2 v x_{0 T_{1}} q_{0 \xi}\right) d \xi=2 k x_{0 T_{1}} \int_{-\infty}^{\infty}\left(q_{0} q_{0 \xi}\right) d \xi=0 .
$$

The above results indicate that the solvability conditions at this order, $\mathcal{O}(\epsilon)$, are always satisfied, regardless of the specific form of the soliton parameters $x_{0}$ and $\sigma_{0}$. Thus, we may proceed by solving Eqs. (15)-(17) (see details for the derivation of these solutions in the Appendix), and find the following exact solutions for the soliton correction $q_{1}$ :

$$
\begin{aligned}
q_{1}^{(\mathrm{i})} & =\frac{3}{2 g \sqrt{d}} x_{0 T_{1}} \xi \operatorname{sech}^{2}(\xi) \\
q_{1}^{(\mathrm{r})} & =-\frac{3 q}{16 g} \sqrt{\frac{d}{2 \nu}} \sigma_{0 T_{1}} \operatorname{sech}^{3}(\xi)[-9 \cosh (\xi) \\
& +\cosh (3 \xi)+12 \xi \sinh (\xi)]
\end{aligned}
$$

Notice that $q_{1}^{(\mathrm{r})} \rightarrow-\frac{3 q}{16 g} \sqrt{\frac{d}{2 \nu}} \sigma_{0 T_{1}}$ as $|\xi| \rightarrow \infty$, a fact that is associated with the emergence of a shelf, i.e., a linear wave adjacent to the soliton. Shelves were first found in the context of perturbed Korteweg-de Vries (KdV) equations 41], and later were also studied for both focusing [44] and defocusing [45] NLS models with a local nonlinearity. Generally, the emergence of shelves lead to the breakdown of the perturbation theory at a higher order approximation in the perturbation scheme [41]. While this issue, along with the appearance of the shelf, are interesting by themselves, they will not be considered here; in our case, the instability induced by the presence of $\sigma_{0 T_{1}}$ in Eq. (28) (see below) plays the dominant role in the evolution of the soliton.

To proceed further, we apply the solvability condition at $\mathcal{O}\left(\epsilon^{2}\right)$, in which case Eqs. (23) and (24) respectively read:

$$
\begin{aligned}
& \int_{-\infty}^{\infty}\left[q _ { 0 \xi } \left(d k x_{0 Y_{1} Y_{1}} q_{0 \xi}+2 \omega \sigma_{0 T_{2}} q_{0}\right.\right. \\
& \left.\left.+2 q_{1 T_{1}}^{(\mathrm{i})}\right)+\theta_{0}\left(\nu k x_{0 Y_{1} Y_{1}} \eta_{0 \xi}\right)\right] d \xi=0 \\
& \Rightarrow x_{0 T_{1} T_{1}}-\frac{3 d^{2} q}{5 \nu} x_{0 Y_{1} Y_{1}}=0,
\end{aligned}
$$

and

$$
\begin{gathered}
\int_{-\infty}^{\infty}\left[q_{0}\left(-d \omega \sigma_{0 Y_{1} Y_{1}} q_{0}+2 k x_{0 T_{2}} q_{0 \xi}-2 q_{1 T_{1}}^{(\mathrm{r})}\right)\right] d \xi=0 \\
\Rightarrow \sigma_{0 T_{1} T_{1}}+\frac{4 d^{2} q}{3 \nu} \sigma_{0 Y_{1} Y_{1}}=0
\end{gathered}
$$

The set of Eqs. (29) and (30), which is one of the the main results of our analytical approach, must be satisfied in order for our original system of Eqs. (15)-(17) to be solvable, up to the order $\mathcal{O}\left(\epsilon^{2}\right)$. We note that, in the above equations, no nonlinear terms in $x_{0}$ [in (29)] and $\sigma_{0}$ [in (30)] are involved, since such terms vanish when applying the solvability condition; thus, Eqs. (29) and (30) do not involve any approximation, up to this order of approximation.

Evidently, Eq. (29) is a hyperbolic PDE (having the form of the usual 2nd-order wave equation) and, thus, its solutions corresponding to bounded initial data never blow up. On the contrary, Eq. (30) is an elliptic PDE (of the Laplace type) and, thus, any bounded initial condition features an exponential growth. As a consequence, the exponential growth of $\sigma_{0}$ will result in an exponential growth of $q_{1}^{(\mathrm{r})}$ as indicated by Eq. (28); in other words, any initial condition of the form (10)-(11) is unstable in the $2 \mathrm{D}$ setting. Notice that the fact that $x_{0}\left(Y_{1}, T_{1}\right)$ obeys a hyperbolic PDE, while $\sigma_{0}\left(Y_{1}, T_{1}\right)$ obeys an elliptic PDE, bears resemblance to the case of the instability of bright soliton stripes of the elliptic NLS equation, with local cubic nonlinearity, in $(2+1)$-dimensions 9 , 41].

\section{Instability and instability growth rate}

To investigate the instability-induced soliton dynamics, first we note that, in practice, the instability is anticipated to manifest itself at finite time. This means that there exists a characteristic timescale $\tau$ for the manifestation of the instability leading the bright soliton stripe to decay into purely 2D structures (similarly to the case of the elliptic 2D NLS 9] ). To calculate this timescale, we need to consider some specific initial conditions for the PDEs (29) and (30). In particular, without loss of generality, we supplement Eq. (29) with the following initial data:

$$
x_{0}\left(0, Y_{1}\right)=\delta \cos \left(K Y_{1}\right), \quad x_{0 T_{1}}\left(0, Y_{1}\right)=0,
$$

and Eq. (30) with the initial data:

$$
\sigma_{0}\left(0, Y_{1}\right)=\delta \cos \left(K Y_{1}\right), \quad \sigma_{0 T_{1}}\left(0, Y_{1}\right)=0,
$$

where $\delta$ and $K$ represent the perturbation amplitude and wavenumber, respectively. Then, the solutions of Eqs. (29)-30 take, respectively, the following form:

$$
\begin{aligned}
x_{0}\left(T_{1}, Y_{1}\right) & =\frac{\delta}{2}\left\{\cos \left[K\left(Y_{1}-\sqrt{\frac{3 d^{2} q}{5 \nu}} T_{1}\right)\right]\right. \\
& \left.+\cos \left[K\left(Y_{1}+\sqrt{\frac{3 d^{2} q}{5 \nu}} T_{1}\right)\right]\right\} \\
\sigma_{0}\left(T_{1}, Y_{1}\right) & =\frac{\delta}{2}\left[\exp \left(K \sqrt{\frac{4 d^{2} q}{3 \nu}} T_{1}\right) \cos \left(K Y_{1}\right)\right. \\
& \left.+\exp \left(-K \sqrt{\frac{4 d^{2} q}{3 \nu}} T_{1}\right) \cos \left(K Y_{1}\right)\right] .
\end{aligned}
$$


Obviously, Eq. (33) represents the usual D'Alembert solution composed by a right- and a left-going wave; this solution is always bounded and never grows. This, however, is not the case of the solution (34), which grows exponentially. In fact, it can be inferred from Eq. (34) that the solution grows in time as: $\sigma_{0} \propto \exp (t / \tau)$, where the characteristic time scale $\tau$ is given by:

$$
\tau=\frac{1}{\Gamma} \equiv \frac{1}{\epsilon K \sqrt{\frac{4 d^{2} q}{3 \nu}}}=\frac{1}{2 \epsilon K d} \sqrt{\frac{3 \nu}{q}},
$$

with $\Gamma=1 / \tau$ being the instability growth rate. It is important to point out that Eq. (35) reveals that, for fixed $\epsilon$, $K, d$ and $q$, the characteristic time $\tau$ scales according to the $\sqrt{\nu}$ law, meaning that the instability manifests itself for longer times as the nolocality becomes stronger. As mentioned in the Introduction, suppression of instabilities is a generic feature of nonlocality; this occurs in our case as well, but the transverse instability of the bright soliton stripe cannot be completely arrested. Nevertheless, strong nonlocality (i.e., large $\nu$ ) is able to significantly prolong the soliton lifetime.

To further quantify the above results, and as a preamble for our numerical simulations, it is now convenient to write down the soliton solution, up to $\mathcal{O}(\epsilon)$, namely:

$$
u(x, y, t)=u_{0}(\xi)+\epsilon u_{1}\left(\xi, T_{1}, Y_{1}\right)+\mathcal{O}\left(\epsilon^{2}\right),
$$

with $\xi=k\left[x-x_{0}\left(T_{1}, Y_{1}\right)\right]$. Substituting Eq. (27), Eq. (28) and Eq. (10) into the expression above the soliton solution takes the form:

$$
\begin{aligned}
u(x, y, t) & =\left\{\frac{3 q}{2 g} \sqrt{\frac{d}{2 \nu}} \operatorname{sech}^{2}(\xi)+\epsilon\left[-\frac{3 q}{16 g} \sqrt{\frac{d}{2 \nu}}\right.\right. \\
& \times \sigma_{0 T_{1}} \operatorname{sech}^{3}(\xi)(-9 \cosh (\xi)+\cosh (3 \xi) \\
& \left.\left.+12 \xi \sinh (\xi))+i \frac{3}{2 g \sqrt{d}} x_{0 T_{1}} \xi \operatorname{sech}^{2}(\xi)\right]\right\} \\
& \times \exp \left[i \omega\left(t+\sigma_{0}\right)\right]+\mathcal{O}\left(\epsilon^{2}\right),
\end{aligned}
$$

with $\omega$ given in Eq. (77). It is now clear that the soliton solution $u(x, y, t)$ grows exponentially due to the presence of the term $\sigma_{0 T_{1}}$ and eventually will break up. In the next Section, we will present numerical results to study the instability dynamics, check the validity of the solution (36), as well as the estimation for the growth rate [Eq. 350] against direct numerical simulations.

\section{NUMERICAL RESULTS}

We now proceed with results obtained by means of dynamical simulations of the system's evolution. The latter are performed by numerically integrating Eqs. (11)-(2) using a high accuracy spectral method [46]. The initial condition is borrowed from the soliton solution of Eq. (36), for $t=0$, using the initial conditions of Eqs. (31)-(32).
In particular, the initial condition for the field $u$ is taken to be:

$$
\begin{aligned}
u(x, y, 0) & =\left\{\frac{3 q}{2 g} \sqrt{\frac{d}{2 \nu}} \operatorname{sech}^{2}[k(x-\delta \cos (\epsilon K y))]\right\} \\
& \times \exp [i \omega \delta \cos (\epsilon K y)] .
\end{aligned}
$$

It is clear that the terms $\propto \delta$ above describe small perturbations in the initial soliton center position and phase, while the argument of the soliton's phase, which is $\propto \epsilon$, implies that the relevant perturbation is a longwavelength one.

The parameter values used in the simulations are:

$$
q=d=g=1, \quad K=3, \quad \epsilon=\delta=0.1
$$

while the nonlocality parameter $\nu$ was varied in the interval $[1,20]$. Notice that both $\epsilon$ and $\delta$, which were considered as small parameters in our perturbation scheme, were assumed to take relatively large values; nevertheless, as we will see, even for such a choice, the analytical results are found to be in good agreement with the results of the simulations. It is also noticed that other choices for the rest of the parameter values led to results qualitatively similar to the ones that will be presented below.

First, we present results showcasing the instabilityinduced dynamics of solitons. In Fig. 1, we show contour plots depicting the evolution of the soliton modulus, $|u(x, y, t)|$, for different times. In the left panels we use the value of the nonlocality parameter $\nu=1$, while in the right panels we showcase the larger nonlocality strength of $\nu=10$; other parameter values are given in Eq. (38). The top panels, (a) and (b), of this figure show the initial condition $(t=0)$, as given in Eq. (37), while the other panels show characteristic snapshots of $u$ for $t \neq 0$; observe that for the weaker nonlocality $(\nu=1)$, the soliton width, $1 / k$ [with $k$ given in Eq. (99)], is shorter.

As is clearly seen, in both cases, the soliton stripes are prone to the instability, which is of the necking type [7]. Nevertheless, the soliton in the setting with $\nu=10$ (right panels), takes a longer time to break up. Hence, nonlocality leads to a substantial suppression of the transverse instability of the soliton stripes, similarly to what was found for the branch of solutions arising from the standard local NLS soliton of $\nu=0$ in Ref. [24]. On the other hand, it is seen that eventually, in either case, the solitons decay into a chain of 2D localized structures, as is also shown in Fig. 2 there, the modulus and the phase of such a chain is depicted at $t=80$, for a nonlocality parameter of $\nu=10$. Notice that the phase profile of the emerging 2D structures, depicted in the right panel of the figure, show that these waveforms are long-lived, vorticity-free ones.

At this point, it is also relevant to test the validity of the analytical estimation for the growth rate $\Gamma=1 / \tau$, with $\tau$ given by Eq. (35). To do this, in Fig. 3, we show the logarithm of the modulus of the difference

$$
D(t)=\log \left|u_{\text {num }}(0,0, t)-u_{0}(0,0, t)\right|,
$$



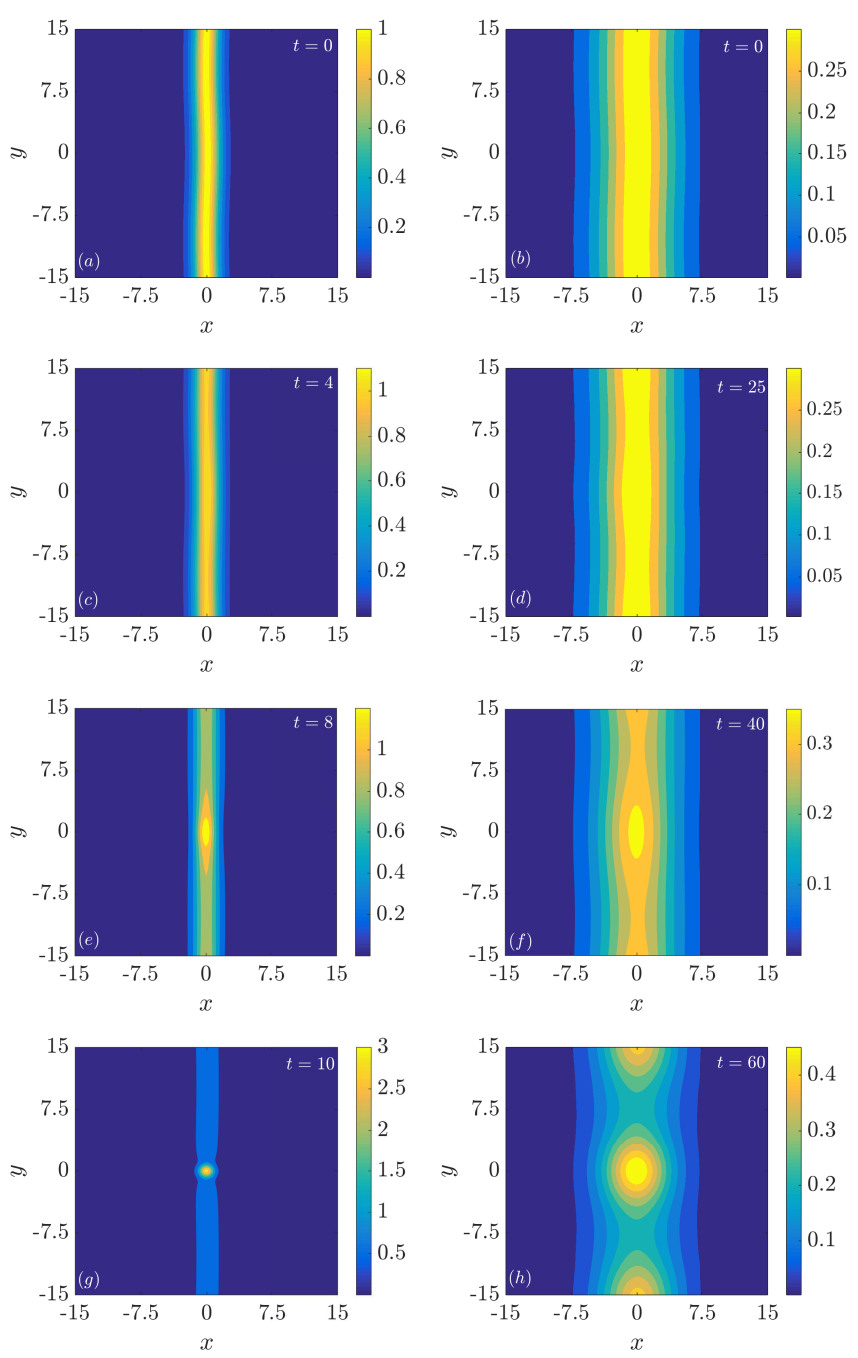

FIG. 1: (Color online) Contour plots showing the evolution of the soliton modulus, $|u(x, y, t)|$, for $\nu=1$ (left panels) and $\nu=10$ (right panels); in addition, $\delta=0.1$, and other parameter values are given in Eq. (38). The panels (a) and (e) show the initial condition [Eq. (37) for $t=0$ ], while the other panels show characteristic snapshots of $u$. Eventually, the soliton decays into a chain of $2 \mathrm{D}$ structures (see also Fig. 2).

where $u_{\text {num }}$ is the numerical solution and $u_{0}$ is the exact analytical soliton solution [see Eq. [10)], evaluated at $x=0, y=0$, as a function of time; shown are curves corresponding to different nonlocality parameters, namely $\nu=1, \nu=5, \nu=10, \nu=15, \nu=20$. The idea here is that, subtracting the exact soliton solution from the numerical one, one seeks to isolate the predicted exponential growth of the soliton correction, and investigate whether it agrees with the analytical prediction of $\exp (t / \tau)$ dependence.

The numerical results, depicted by the dashed curves, show that at the early stage of the evolution $(t \lesssim 2$ for $\nu=1$ up to $t \lesssim 7$ for $\nu=20$ ), the considered function undergoes a transient stage until the instability gets activated. Once the latter activation materializes, the rele-
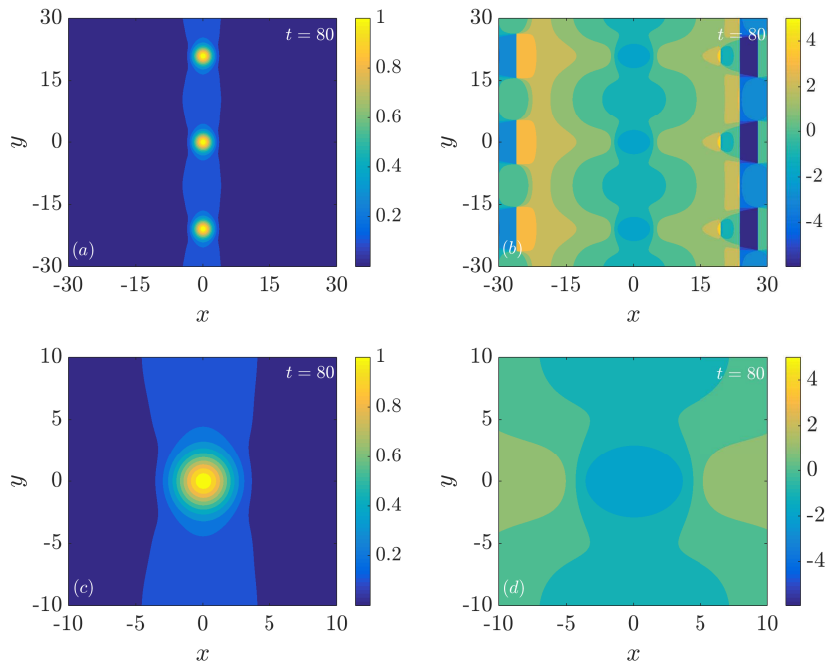

FIG. 2: (Color online) Contour plots showing the modulus [panel (a)] and the phase [panel (b)] of $u(x, y, t)$ at $t=80$, for nonlocality parameter $\nu=10$; other parameters are kept fixed, as given in Eq. (38). This state consists of a chain of vorticity-free $2 \mathrm{D}$ structures, as is also seen in the bottom zoom [panels (c) and (d)].

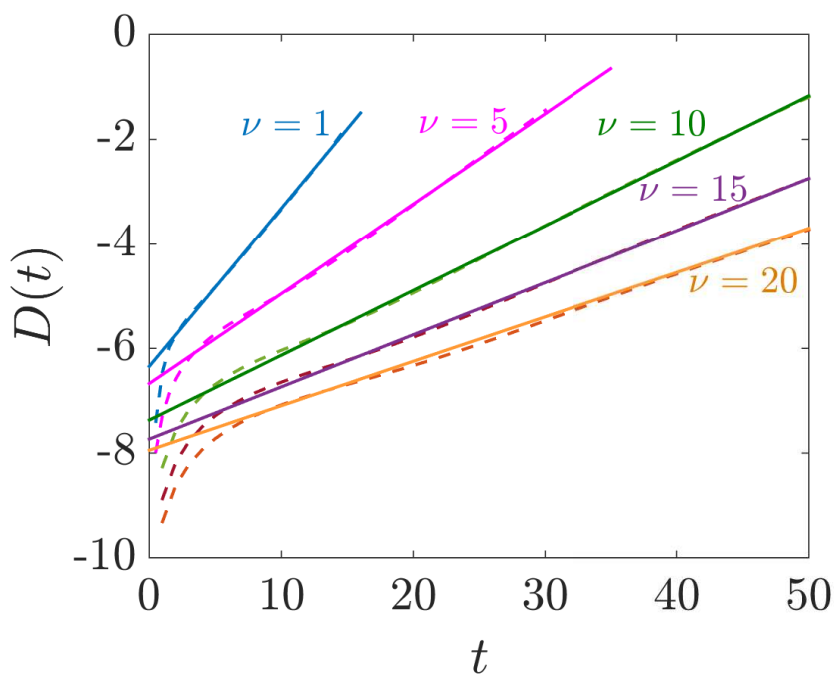

FIG. 3: (Color online) The logarithm of the modulus of the difference $D(t)=\log \left|u_{\text {num }}(0,0, t)-u_{0}(0,0, t)\right|$, as a function of time, for different values of the nonlocal parameter $\nu$; here, $u_{\text {num }}$ is the numerical solution, and $u_{0}$ is the exact analytical soliton solution. The dashed lines correspond to the numerical results for each value of $\nu$, while the solid lines to their corresponding linear fits (once the instability sets in and, indeed, after an initial transient stage). The latter are in good agreement with the predicted growth rates of Eq. (35) (see Table I).

vant plot of the logarithmic diagnostic of choice features a linear growth. This is obviously a signature of the exponential growth of the solution that was predicted above, 

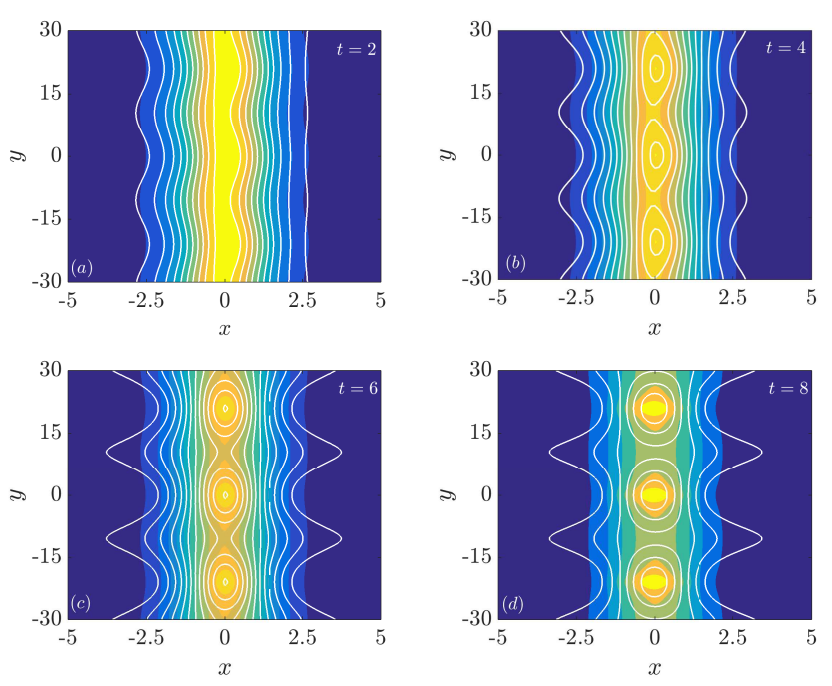

FIG. 4: (Color online) Solid (white) iso-contour lines of constant modulus, corresponding to the analytical solution (36), are "superimposed" on top of contour plots showing the modulus of the numerical solution, for $t=2,4,6,8$, and for $\nu=1$.

while the slopes of the pertinent straight lines should correspond to the growth rates for the different values of $\nu$ [see Eq. (35)]. Indeed, the slopes of the relevant linear fits (solid lines) are close to the analytically predicted growth rates $\Gamma=1 / \tau$ for each value of $\nu$, as shown in Table I. As seen in the table, the resulting relative error between the numerical result and the analytical prediction ranges between $8 \%$ to $12 \%$, for all considered values of $\nu$, signaling the good agreement between the two.

\begin{tabular}{cccc}
\hline \hline$\nu$ & Linear fit slope & $\Gamma=1 / \tau$ & Approximate \% error \\
\hline 1 & 0.38 & 0.35 & 8 \\
5 & 0.17 & 0.15 & 10 \\
10 & 0.12 & 0.11 & 9 \\
15 & 0.10 & 0.09 & 10 \\
20 & 0.09 & 0.08 & 12 \\
\hline
\end{tabular}

TABLE I: Comparison between the slopes of the linear fits of the numerical data of Fig. 3 and the analytical prediction for the growth rate, $\Gamma=1 / \tau$, for various values of $\nu$.

It is also relevant to provide an additional test for the validity of our analytical result concerning the solution given in Eq. (36), against results of the numerical simulations. In Fig. 4, solid (white) iso-contour lines of constant modulus, corresponding to the approximate analytical solution (36), are "superimposed" on top of contour plots showing the modulus of the numerical solution, for $\nu=1$. In all panels, (a)-(d), corresponding to $t=2,4,6,8$, a qualitative agreement between the numerical and the analytical solution is observed, especially around the soliton maximum. Naturally, discrepancies occur at the soliton tails as contributions beyond our analysis of $\mathcal{O}(\epsilon)$ (such as, e.g., ones at $\mathcal{O}\left(\epsilon^{2}\right)$ ) become progressively more important. Notice that the discrep- ancy between the numerical and the analytical solution becomes larger as time increases, due to the exponential growth of the instability, which is only captured via the $\mathcal{O}(\epsilon)$ terms in the approximate analytical solution of Eq. (36). Finally, and as is naturally expected, past the time $t=8$ [panel $(\mathrm{d})]$, the analytical result fails, as the soliton has already been destroyed.

\section{CONCLUSIONS}

In this work, we studied the transverse dynamics and, in particular, the transverse instability of bright soliton stripes in media with a spatially nonlocal nonlinear response. The considered nonlocal nonlinear Schrödinger (NLS) model describes beam propagation in different types of nonlocal nonlinear media, including thermal media, plasmas, and nematic liquid crystals.

Starting with an exact 1D bright soliton solution of the system (which, however, had no analogue in the (local) case of nonlocality parameter $\nu=0$ ), we employed a direct multiscale perturbation method to study the transverse dynamics of solitons. Assuming that the soliton's center $x_{0}$ and phase $\sigma_{0}$ become functions of a slow time $T_{1}=\epsilon t$ and a slow transverse coordinate $Y_{1}=\epsilon y$ (with $0<\epsilon \ll 1$ ), we found the following. First, $x_{0}$ and $\sigma_{0}$ obey, respectively, a hyperbolic and an elliptic 2nd-order PDE (with respect to $T_{1}$ and $Y_{1}$ ), namely a 2 nd-order wave equation and a Laplace-type equation. The solution of these evolution equations, together with the solution for the 1st-order correction to the soliton shape, led to an approximate solution of the original nonlocal NLS model, valid up to $\mathcal{O}(\epsilon)$. It was found that the transverse instability, caused by the exponential growth of the phase $\sigma_{0}$, is of the necking type, and leads to the break up of solitons. The instability growth rate was found to scale with the nonlocality parameter $\nu$ according to the law $1 / \sqrt{\nu}$. This fact indicates the nonlocality-induced suppression (but not full arrest) of the transverse instability of the bright soliton stripes, in line with results for different solitonic structures (bearing a $\nu=0$ limit) within the model, as reported in previous works [24].

Direct numerical simulations were found to be in good agreement with the analytical predictions. As concerns the analytically found instability growth rate, it was shown that it is in good agreement with the numerical one (past an initial transient stage), for values of the nonlocality parameter in the interval $1 \leq \nu \leq 20$. In fact, the relative percentage error between pertinent analytical and numerical results was found to be around $10 \%$ for all the cases that were considered. In addition, the approximate analytical soliton solution [valid up to $\mathcal{O}(\epsilon)$ ] was found to follow the numerical one, with the agreement between the two being better near the the soliton center. The discrepancy between the two, especially near the soliton tails and at later times, was attributed to the fact that our analytical approximation cannot capture higher-order effects [of order $\mathcal{O}\left(\epsilon^{j}\right)$, with $j \geq 2$ ], and it 
completely fails after the initial solitonic stripe deforms into a sequence of two-dimensional (non-vortical) solitonic "blobs".

Our work paves the way for interesting future studies. For instance, our perturbative approach could also be applied in the case of a defocusing nonlocal nonlinearity, which supports dark solitons, both in 1D [2932] and in 2D [47 49]. In such a defocusing setting, it would be interesting to study analytically the suppression of the transverse (snaking) instability of dark soliton stripes (see relevant numerical results in Ref. [33]). Furthermore, the analytical study of the transverse dynamics of solitons in multicomponent nonlocal systems (see, e.g., Ref. [50]) is another interesting and relevant theme. This is due to the fact that that there exists a plethora of vector solitons in such settings [51 53], while studies on the transverse dynamics of solitons are mainly numerical ones [54]. It would, therefore, be particularly interesting to investigate the combined effect of nonlocality and soliton coupling on the soliton instability dynamics. Such studies are in progress and relevant results will be reported elsewhere.

\section{Appendix A: Solution of $\mathcal{O}(\epsilon)$ perturbation equations}

Here, we provide a solution of the system of Eqs. (15)(17), at the order $\mathcal{O}(\epsilon)$ (i.e., for $j=1$ ). As is observed, Eq. (16) is decoupled from Eqs. (15) and (17) and can be solved separately. Having found the solution of the homogeneous equation, $q_{1 h}^{(\mathrm{i})}=q_{0}$ [see Eq. (22)], we seek for the solution of the full, inhomogeneous, equation in the form:

$$
q_{1}^{(\mathrm{i})}\left(\xi, T_{1}, Y_{1}\right)=q_{0}(\xi) f\left(\xi, T_{1}, Y_{1}\right),
$$

where $f(\xi)$ is an unknown function, to be determined. Substituting Eq. (A1) into Eq. (16), and employing the reduction of order method, we find:

$$
\begin{aligned}
& q_{1}^{(\mathrm{i})}=q_{0} {\left[\int \frac{1}{q_{0}^{2}}\left(\int \frac{1}{k^{2} d} q_{0} F_{1}^{(\mathrm{i})} d \xi\right) d \xi\right.} \\
&\left.+\int \frac{A_{1}\left(T_{1}, Y_{1}\right)}{q_{0}^{2}} d \xi+A_{2}\left(T_{1}, Y_{1}\right)\right]
\end{aligned}
$$

where $A_{1}$ and $A_{2}$ are unknown functions of the slow variables $T_{1}$ and $Y_{1}$. Next, imposing the boundary condition $q_{1}^{(\mathrm{i})} \rightarrow 0$ as $\xi \rightarrow \pm \infty$, we obtain $A_{1}\left(T_{i}, Y_{i}\right)=0$ and we choose, without loss of generality, $A_{2}\left(T_{i}, Y_{i}\right)=0$ too; indeed, the term involving $A_{2}$ is of the form $\epsilon A_{2}\left(T_{i}, Y_{i}\right) q_{0}$ in the asymptotic expansion and can be absorbed in the $\mathcal{O}(1)$ solution. This way, upon performing the relevant integrations, we derive from Eq. (A2) the solution (27).
The next step is to solve the system of Eqs. (15) and (17). To do so, first we solve Eq. (17) for the field $\theta_{1}$, and find:

$$
\begin{aligned}
\theta_{1}=\frac{1}{6 g} \sqrt{\frac{d}{2 \nu}} & {\left[3 q \sqrt{\frac{2 d}{\nu}} \sigma_{0 T_{1}}+\left(-6 g+4 g \cosh ^{2}(\xi)\right) q_{1}^{(\mathrm{r})}\right.} \\
& \left.-g \cosh ^{2}(\xi) q_{1 \xi \xi}^{(\mathrm{r})}\right],
\end{aligned}
$$

where we have substituted the expression $q_{0}$ from Eq. (9). Obviously, once $q_{1}^{(\mathrm{r})}$ is found (see below), Eq. (A3) can be used for the determination of $\theta_{1}$.

Next, we substitute Eq. (A3) into Eq. (15), and using the expressions for $\theta_{0}$ and $q_{0}$ from Eqs. (11) and Eqs. (9), we find the following 4 th-order ODE for $q_{1}^{(\mathrm{r})}$ :

$$
\begin{aligned}
q_{1 \xi \xi \xi \xi}^{(\mathrm{r})} & +4 \tanh (\xi) q_{1 \xi \xi \xi}^{(\mathrm{r})}-4\left(1-\operatorname{sech}^{2}(\xi)\right) q_{1 \xi \xi}^{(\mathrm{r})} \\
& -16 \tanh (\xi) q_{1 \xi}^{(r)}-\left(16 \operatorname{sech}^{2}(\xi)+72 \operatorname{sech}^{4}(\xi)\right) q_{1}^{(\mathrm{r})} \\
& +\frac{12 q}{g} \sqrt{\frac{2 d}{\nu}} \sigma_{0 T 1} \operatorname{sech}^{2}(\xi)=0
\end{aligned}
$$

To solve the above equation, first we note that a homogeneous solution of Eq. (A4) is $q_{0 \xi}$ [see Eq. (22)]. Furthermore, we can deduce that via a variation of constants method that $q_{0 \xi} \int\left(1 / q_{0 \xi}^{2}\right) d \xi$ is another homogeneous solution of Eq. A4. Having at hand two homogeneous solutions, we introduce the following transformation:

$$
\begin{aligned}
q_{1}^{(\mathrm{r})}(\xi) & =\left(q_{0 \xi} \int \frac{1}{q_{0 \xi}^{2}} d \xi\right)\left(\int q_{0 \xi} w(\xi) d \xi\right) \\
& -q_{0 \xi} \int\left(q_{0 \xi} \int \frac{1}{q_{0 \xi}^{2}} d \xi\right) w(\xi) d \xi
\end{aligned}
$$

where $w(\xi)$ is an unknown function to be determined. Substituting Eq. (A5) into Eq. (A4) we obtain the following 2nd-order ODE for $w(\xi)$ :

$$
\begin{aligned}
w_{\xi \xi} & +4 \tanh (\xi) w_{\xi}-8 \operatorname{sech}^{2}(\xi) w \\
& +\frac{12 q}{g} \sqrt{\frac{2 d}{\nu}} \sigma_{0 T_{1}} \operatorname{sech}^{2}(\xi)=0
\end{aligned}
$$

It is easy to check that a partial solution of Eq. (A6) is:

$$
w(\xi)=\frac{3 q}{2 g} \sqrt{\frac{2 d}{\nu}} \sigma_{0 T_{1}} .
$$

Finally, substituting Eq. (A7) back to Eq. (A5), we derive the solution for $q_{1}^{(r)}(\xi)$, namely Eq. (28).
[1] E. Infeld and G. Rowlands. Nonlinear Waves, Solitons and Chaos. Cambridge University Press, 1990.
[2] V. E. Zakharov and L. A. Ostrovsky. Modulation insta- 
bility: The beginning. Physica D, 238:540-548, 2009.

[3] V. E. Zakharov and A. M. Rubenchik. Instability of waveguides and solitons in nonlinear media. Sov. Phys. JETP, 38:494-500, 1974.

[4] S. J. Han. Stability of envelope waves. Phys. Rev. A, 20:2568-2573, 1979.

[5] E. A. Kuznetsov and S. K. Turitsyn. Instability and collapse of solitons in media with a defocusing nonlinearity. J. Exp. Theor. Phys., 67:1583-1588, 1988.

[6] D. E. Pelinovsky, Yu. A. Stepanyants, and Yu. S. Kivshar. Self-focusing of plane dark solitons in nonlinear defocusing media. Phys. Rev. E, 51:5016-5026, 1995.

[7] E. A. Kuznetsov, A. M. Rubenchik, and V. E. Zakharov. Soliton stability in plasmas and hydrodynamics. Phys. Rep., 142:103-165, 1986.

[8] Yu. S. Kivshar and D. E. Pelinovsky. Self-focusing and transverse instabilities of solitary waves. Phys. Rep., 331:117-195, 2000.

[9] J. Yang. Nonlinear Waves in Integrable and Nonintegrable Systems. SIAM, 2010.

[10] B. P. Anderson, P. C. Haljan, C. A. Regal, D. L. Feder, L. A. Collins, C. W. Clark, and E. A. Cornell. Watching dark solitons decay into vortex rings in a bose-einstein condensate. Phys. Rev. Lett., 86:2926-2929, Apr 2001.

[11] S.-P. Gorza, B. Deconinck, Ph. Emplit, T. Trogdon, and M. Haelterman. Experimental demonstration of the oscillatory snake instability of the bright soliton of the $(2+1) \mathbf{D}$ hyperbolic nonlinear schrödinger equation. Phys. Rev. Lett., 106:094101, Mar 2011.

[12] Simon-Pierre Gorza, Bernard Deconinck, Thomas Trogdon, Philippe Emplit, and Marc Haelterman. Neck instability of bright solitons in normally dispersive kerr media. Opt. Lett., 37(22):4657-4659, Nov 2012.

[13] Z. H. Musslimani, M. Segev, A. Nepomnyashchy, and Yu. S. Kivshar. Suppression of transverse instabilities for vector solitons. Phys. Rev. E, 60:R1170-R1173, 1999.

[14] Z. H. Musslimani and J. Yang. Transverse instability of strongly coupled dark-bright manakov vector solitons. Opt. Lett., 26:1981-1983, 2001.

[15] C. Anastassiou, M. Soljacic, M. Segev, E. D. Eugenieva, D. N. Christodoulides, D. Kip, Z. H. Musslimani, and J. P. Torres. Eliminating the transverse instabilities of kerr solitons. Phys. Rev. Lett., 85:4888-4891, 2000.

[16] J. Yang. Transversely stable soliton trains in photonic lattice. Phys. Rev. A, 84:033840, 2011.

[17] J. Yang, D. Gallardo, A. Miller, and Z. Chen. Elimination of transverse instability in stripe solitons by onedimensional lattices. Opt. Lett., 37:1571-1573, 2012.

[18] Manjun Ma, R. Carretero-González, P. G. Kevrekidis, D. J. Frantzeskakis, and B. A. Malomed. Controlling the transverse instability of dark solitons and nucleation of vortices by a potential barrier. Phys. Rev. A, 82:023621, 2010.

[19] A. G. Litvak, V. A. Mironov, G. M. Fraiman, and A. D. Yunakovskii. Thermal self-effect of wave beams in a plasma with a nonlocal nonlinearity. Sov. J. Plasma Phys., 1:60-71, 1975.

[20] D. Suter and T. Blasberg. Stabilization of transverse solitary waves by a nonlocal response of the nonlinear medium. Phys. Rev. A, 48:4583-4587, 1993.

[21] C. Rotschild, T. Carmon, O. Cohen, O. Manela, and M. Segev. Solitons in nonlinear media with an infinite range of nonlocality: first observation of coherent elliptic solitons and of vortex-ring solitons. Phys. Rev. Lett.,
95:213904, 2005.

[22] C. Conti, M. Peccianti, and G. Assanto. Route to nonlocality and observation of accessible solitons. Phys. Rev. Lett., 91:073901, 2003.

[23] P. Pedri and L. Santos. Two-dimensional bright solitons in dipolar Bose-Einstein condensates. Phys. Rev. Lett., 95:200404, 2005.

[24] YuanYao Lin, Ray-Kuang Lee, and Yu. S. Kivshar. Suppression of soliton transverse instabilities in nonlocal nonlinear media. J. Opt. Soc. Am. B, 2008:576-581, 2008.

[25] S. K. Turitsyn. Spatial dispersion of nonlinearity and stability of many dimensional solitons. Theor. Math. Phys., 64:797-801, 1985.

[26] W. Krolikowski, O. Bang, N. I. Nikolov, D. Neshev, J. Wyller, J. J. Rasmussen, and D. Edmundson. Modulational instability, solitons and beam propagation in spatially nonlocal nonlinear media. J. Opt. B: Quantum Semiclass. Opt., 6:S288-S294, 2004.

[27] D. Mihalache, D. Mazilu, F. Lederer, B. A. Malomed, Y. V. Kartashov, L.-C. Crasovan, and L. Torner. Threedimensional spatiotemporal optical solitons in nonlocal nonlinear media. Phys. Rev. E, 73:025601(R), 2006.

[28] D. Mihalache. Multidimensional solitons and vortices in nonlocal nonlinear optical media. Rom. Rep. Phys., 59:515-522, 2007.

[29] A. Dreischuh, D. N. Neshev, D. E. Petersen, O. Bang, and W. Krolikowski. Observation of attraction between dark solitons. Phys. Rev. Lett., 96:043901, 2006.

[30] Y. V. Kartashov and L. Torner. Gray spatial solitons in nonlocal nonlinear media. Opt. Lett., 32:946-948, 2007.

[31] A. Piccardi, A. Alberucci, N. Tabiryan, and G. Assanto. Dark nematicons. Opt. Lett., 36:1356-1358, 2011.

[32] T. P. Horikis. Small-amplitude defocusing nematicons. J. Phys. A: Math. Theor., 48:02FT01, 2015.

[33] A. Armaroli and S. Trillo. Suppression of transverse instabilities of dark solitons and their dispersive shock waves. Phys. Rev. A, 80:053803, 2009.

[34] N. Ghofraniha, C. Conti, G. Ruocco, and S. Trillo. Shocks in nonlocal media. Phys. Rev. Lett., 99:043903, 2007.

[35] C. Conti, A. Fratalocchi, M. Peccianti, G. Ruocco, and S. Trillo. Observation of a gradient catastrophe generating solitons. Phys. Rev. Lett., 102:083902, 2009.

[36] A. G. Litvak, V. A. Mironov, G. M. Fraiman, and A. D. Yunakovskii. Thermal self-effect of wave beams in a plasma with a nonlocal nonlinearity. Sov. J. Plasma Phys., 1:60-71, 1975.

[37] A. I. Yakimenko, Y. A. Zaliznyak, and Yu. S. Kivshar. Stable vortex solitons in nonlocal self-focusing nonlinear media. Phys. Rev. E, 71:065603(R), 2005.

[38] M. Peccianti and G. Assanto. Nematicons. Phys. Rep., 516:147-208, 2012.

[39] A. Alberucci and G. Assanto. Modeling nematicon propagation. Mol. Cryst. Liq. Cryst., 572:2-12, 2013.

[40] M. Z. Pesenson. Nonlinear waves traveling upon a front of solitons. Phys. Fluids A, 3:3001-3006, 1991.

[41] M. J. Ablowitz and H. Segur. Solitons and the Inverse Scattering Transform. SIAM, 1981.

[42] G. Assanto. Nematicons: Spatial Optical Solitons in Nematic Liquid Crystals. New Jersey: Wiley-Blackwell, 2012.

[43] J. M. L. MacNeil, N. F. Smyth, and G. Assanto. Exact and approximate solutions for solitary waves in nematic liquid crystals. Physica D, 284:1-15, 2014. 
[44] W. L. Kath and N. F. Smyth. Soliton evolution and radiation loss for the nonlinear Schrödinger equation. Phys. Rev. E, 51:1484-1492, 1995.

[45] M. J. Ablowitz, S. D. Nixon, T. P. Horikis, and D. J. Frantzeskakis. Perturbations of dark solitons. Proc. $R$. Soc. A, 467:2597-2621, 2011.

[46] A. Kassam and L. N. Trefethen. Fourth-order time stepping for stiff PDEs. SIAM J. Sci. Comput., 26:1214-1233, 2005.

[47] T. P. Horikis and D. J. Frantzeskakis. Asymptotic reductions and solitons of nonlocal nonlinear Schrödinger equations. J. Phys. A: Math. Theor., 49:205202, 2016.

[48] T. P. Horikis and D. J. Frantzeskakis. Ring dark and antidark solitons in nonlocal media. Opt. Lett., 41:583586, 2016.

[49] T. P. Horikis and D. J. Frantzeskakis. Light meets water in nonlocal media: Surface tension in optics. Phys. Rev. Lett., 118:243903, 2017.
[50] A. Alberucci, M. Peccianti, G. Assanto, A. Dyadyusha, and M. Kaczmarek. Two-color vector solitons in nonlocal media. Phys. Rev. Lett., 97:153903, 2006.

[51] Y. Lin and R.-K. Lee. Dark-bright soliton pairs in nonlocal nonlinear media. Opt. Express, 15:8781, 2007.

[52] T. P. Horikis and D. J. Frantzeskakis. Vector nematicons: Coupled spatial solitons in nematic liquid crystals. Phys. Rev. A, 94:053805, 2016.

[53] G. N. Koutsokostas, T. P. Horikis, D. J. Frantzeskakis, B. Prinari, and G. Biondini. Multiscale expansions and vector solitons of a two-dimensional nonlocal nonlinear Schrödinger system. Stud. Appl. Math., 145:739-764, 2020.

[54] G. N. Koutsokostas, T. P. Horikis, D. J. Frantzeskakis, B. Prinari, and G. Biondini. Transverse dynamics of vector solitons in defocusing nonlocal media. Eur. Phys. J. Plus, 135:546, 2020. 